\title{
A Geometric Determination of the Distance to SN 1987A and the LMC
}

\author{
NINO PANAGIA ${ }^{1,2}$ \\ ${ }^{1}$ Space Telescope Science Institute, 3700 San Martin Drive, Baltimore, MD \\ 21218, USA; panagia@stsci.edu \\ ${ }^{2}$ Affiliated with the Space Telescope Division of the European Space Agency, \\ ESTEC, Noordwijk, Netherlands
}

\begin{abstract}
Summary. Using the definitive reductions of the IUE light curves by 14 and an extensive set of HST images of SN 1987A we have repeated and improved our original analysis 8 to derive a better determination of the distance to the supernova. In this way we have obtained an absolute size of the $\operatorname{ring} R_{\mathrm{abs}}=(6.23 \pm 0.08) \times 10^{17} \mathrm{~cm}$ and an angular size $R^{\prime \prime}=808 \pm 17$ mas, which give a distance to the supernova $d(S N 1987 A)=51.4 \pm 1.2 \mathrm{kpc}$ and a distance modulus $(m-M)_{S N 1987 A}=18.55 \pm$ 0.05. Allowing for a displacement of SN 1987A position relative to the LMC center, the distance to the barycenter of the Large Magellanic Cloud is also estimated to be $d(L M C)=51.7 \pm 1.3 \mathrm{kpc}$, which corresponds to a distance modulus of $(m-$ $M)_{L M C}=18.56 \pm 0.05$.
\end{abstract}

\section{Introduction}

Cepheid variables are possibly the most reliable, and certainly the most widely used secondary distance indicators to measure distances up to several tens of Mpc. Because of this they play a crucial role in the determination of the cosmological distance scale (for a review see the proceedings of the STScI Symposium The Extragalactic Distance Scale, 6]). On the other hand, the calibration of Cepheids as distance indicators is based on the study of Cepheid variables in the LMC and, therefore, determining the distance to the Large Magellanic Cloud is a fundamental step in establishing a cosmological distance scale because the zero point of the Cepheid calibration relies crucially on the calibration of the LMC distance.

Various methods have been employed to measure the distance to the LMC, with various degrees of success and/or accuracy (e.g. 7]). All methods, however, are indirect in that they all depend on the calibration of other distance indicators, and, therefore, have only a statistical value. Moreover, different distance indicators appear to give discordant results that are not compatible with each other, thus making the distance issue very slippery. 
The presence of the famous circumstellar ring around SN 1987A has provided a unique opportunity to determine the distance to the LMC directly by using a purely geometric method: it consists in measuring the angular size of the ring from high resolution images and comparing it to the absolute size as estimated from the evolution of emission lines produced by the ring ionized gas (see e.g. [8, 1, 2]).

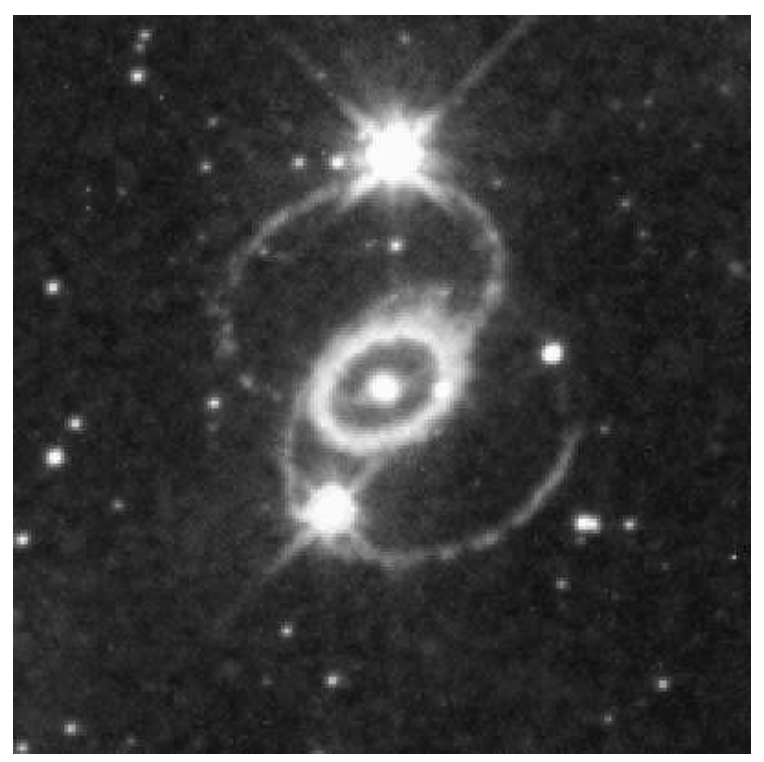

Fig. 1. An $8^{\prime \prime} \times 8^{\prime \prime}$ region centered on SN 1987A as observed on September 24, 1994, with the HST-WFPC2 in an [OIII] $5007 \AA$ filter. In addition to the supernova, this figure shows clearly the presence of the three circumstellar rings, a brighter equatorial ring and two fainter, larger rings that are loosely aligned along the polar axis.

In 1991 Panagia et al. 8] estimated the distance to SN 1987A (51.2 \pm $3.1 \mathrm{kpc}$ ) from a comparison of the angular size of the inner circumstellar ring as measured with the HST-FOC in August 1990 [5], with the ring absolute size as determined from the peaks of its UV emission line light curves.

More recently, Gould re-examined the problem adopting an infinitely narrow ring geometry and retaining Panagia et al. 8] assumption of an exponential law for the line emissivity [2, 3. Thus, using the same data as in Panagia et al. [8] and Sonneborn et al. [14, respectively, but including only the NIV] and NIII] light curves, and adopting the average [OIII] ring size as measured by Plait et al. 10 over the period August 1990 - May 1993, Gould concluded that the distance to SN 1987A be less than $47 \mathrm{kpc}$.

The new reductions of the IUE spectra, done by Sonneborn et al. [14, have produced more accurate and reliable light curves. Therefore, we have decided 
to repeat our analysis using the new data set and including a more accurate and realistic estimate of the ring angular size obtained from the study of an extensive set of HST images of SN 1987A. Here, we present a brief outline of our analysis and the main results of our study. A complete account of this work will be presented in a forthcoming paper [9].

\section{The Angular Size of the Ring}

The inner circumstellar ring is clearly extended with a HPW of about $1 / 7$ its radius (e.g. [5, 9]). The finite width of the ring makes the definition of an average size a very delicate one, which, if done improperly, may introduce errors as large as, say, half the HPW, i.e. as much as $7 \%$ or more.

Also, to derive the distance to SN 1987A one should compare the absolute ring size, determined from the light curves of twice ionized $\mathbf{N}$ and $\mathbf{C}$ and three times ionized $\mathbf{N}$, with the angular size of the ring as measured at the time of the peak for radiation emitted by ions of comparable ionization stages.

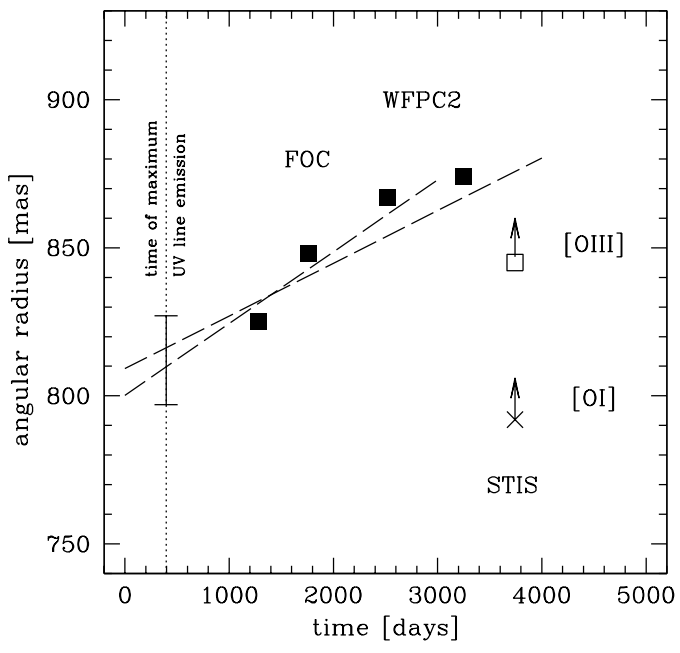

Fig. 2. The surface brightness averaged radius of the ring as measured in $F O C$ and WFPC2 images taken with a narrow band [OIII] filter, and the recent measurements made with the STIS in the light of the [OIII] $5007 \AA$ and [OI] $6300 \AA$ lines 15. The two straight lines are the best fits to the $F O C+W F P C 2$ points only, and to the $F O C+W F P C 2+S T I S[\mathrm{OIII}]$ sizes, respectively. The error bar represents the range of possible values of the radius at the time of the UV maximum. 
While the images obtained with $H S T$ in the [OIII] line filter (see e.g. Fig. 1) satisfy the second requirement (comparable ions), they fail to satisfy the first one (comparable epochs). On the other hand, the analysis of both $F O C$ and $W F P C 2$ images have revealed that the ring angular size, as estimated from [OIII] images, appears to increase with time ([10, 9]; see Fig. 2) while the one derived from $\mathrm{H} \beta$ and [NII] images remains constant in time and virtually coincides with the size measured in the earliest [OIII] image. This is the effect of both cooling and recombination of the OIII ion, that cause the [OIII] $5007 \AA$ line intensity to decline more quickly at the inner edge of the ring where the density is believed to be higher. An experimental confirmation of this effect is provided by HST-STIS imaging-spectroscopy of SN 1987A, obtained in April 1997 [15], that has shown an appreciably smaller ring size in the [OI] $6300 \AA$ line than it is in the [OIII] $5007 \AA$ line.

Therefore, the best value of the ring angular size to compare with the absolute size determined from the UV lines is an extrapolation of the observed sizes, as measured with $H S T$ in the [OIII] $5007 \AA$ filter, back to the epoch of maximum UV line emission (approximately 400 days after the explosion, i.e. around early April 1988; cf. Fig. 31). In this way we obtain:

$$
R^{\prime \prime}=808 \pm 17 \text { mas } .
$$

\section{The Absolute Size of the Ring}

It has been shown 8, 1, 2, that under the assumption of an infinitely narrow width the absolute radius of the ring can be derived from measurements of the onset time of the UV line emission, $t_{\circ}$, and the time of maximum UV line emission $t_{\max }$ because they correspond to the times when the near side and the far side of the ring start shining as a result of the ionization due to the initial UV flash from the supernova explosion. A simple geometric argument gives

$$
R=c\left(t_{\circ}+t_{\max }\right) / 2 .
$$

As mentioned before, one has to measure the absolute size for the same emitting ion for which one can measure the angular size. In addition, one has also to take into account that the ring is clearly extended with a width $\delta R \simeq R / 7$. Therefore, we have limited our analysis to the UV light curves of twice ionized ions, namely OIII, NIII and CIII, and we have compared them to theoretical light curves computed under the following assumptions:

- The ring is circular and has a gaussian width with HPW of $14 \%$ the ring radius.

- The intrinsic emission of each ion decays exponentially with time.

- The free parameters are the radius and the inclination angle of the ring, the specific emissivity at time $t=0$ and the decay time of each line. 

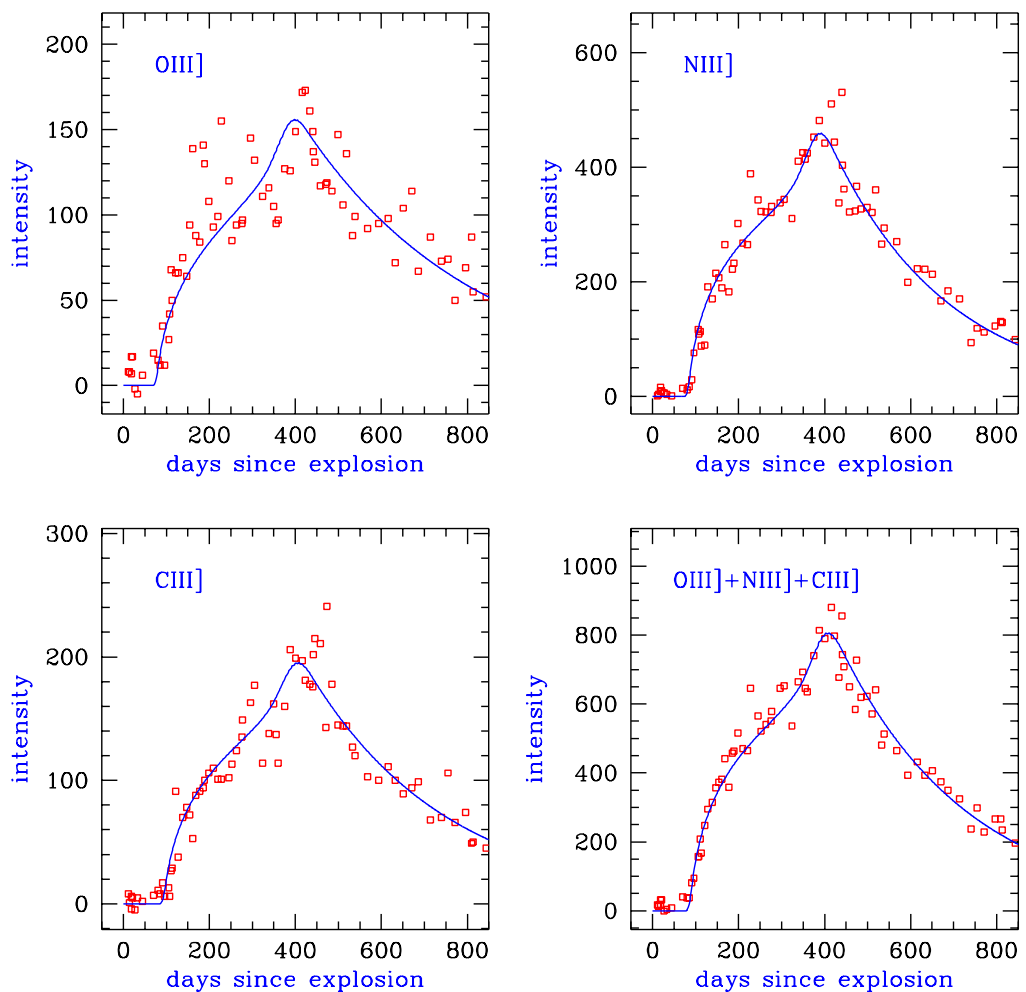

Fig. 3. The observed intensities (in units of $10^{-15} \mathrm{erg} \mathrm{cm}^{-2} \mathrm{~s}^{-1}$ ) of the OIII] $1666 \AA$, NIII] $1750 \AA$, and CIII] $1909 \AA$ lines and their straight sum are presented and compared to their best-fit model light curves.

The best fits to the light curves for the OIII] $1666 \AA$, NIII] $1750 \AA$, and CIII] 1909 A lines are shown in Fig. 3

We also show the composite light curve, sum of the OIII], NIII] and CIII] line intensities, and its best model fit: we note that the scatter in the composite light curve is greatly reduced relative to the three light curves, indicating that most of the fluctuation is actually noise. The individual determinations of the absolute radius fall in the range 230 to 248 light-days, or 6.0 to $6.4 \times 10^{17} \mathrm{~cm}$, with uncertainties of about $4 \%$ for OIII] and CIII], and slightly above $1 \%$ for NIII], resulting in an average value of

$$
R=(6.23 \pm 0.08) \times 10^{17} \mathrm{~cm} .
$$

\section{Distance Determination}

Comparing the absolute size of the circumstellar ring to its angular size, we derive a distance to SN 1987A 
$\mathrm{d}(\mathrm{SN1987A})=\mathbf{5 1 . 4} \pm \mathbf{1 . 2} \mathrm{kpc} \quad(\mathrm{m}-\mathrm{M})_{\mathrm{SN} 1987 \mathrm{~A}}=\mathbf{1 8 . 5 5} \pm \mathbf{0 . 0 5}$.

This value is very close to our original determination [8] but is considerably more accurate. Actually, it could still be an underestimate to the true distance to SN 1987A, because if the ring is not perfectly circular, as hinted by the marginal discrepancy between the inclinations determined from light curve fitting $\left(i \simeq 42^{\circ}\right)$ and from the major to minor axis ratio $\left(i \simeq 44^{\circ}\right)$, then the distance may have to be increased by as much as $2 \%$. 3 .

Allowing for the difference of position of SN 1987A relative to the LMC barycenter [16] the best estimate of the distance to the center of mass of the LMC is found to be

$$
\mathrm{d}(\mathrm{LMC})=51.7 \pm 1.3 \mathrm{kpc} \quad(\mathrm{m}-\mathrm{M})_{\mathrm{LMC}}=18.56 \pm 0.05 .
$$

The error includes the uncertainties on the SN $1987 \mathrm{~A}$ distance $( \pm 1.2 \mathrm{kpc})$ as well as those on the depth toward SN 1987A $( \pm 0.2 \mathrm{kpc})$ and the relative position of the LMC barycenter $( \pm 0.3 \mathrm{kpc})$.

\section{Discussion and Consequences for the Cosmological Distance Scale}

In the literature one finds a number of determinations of SN 1987A distance which are all based on the analysis of UV line light curves and HST imaging but provide values that may be quite discrepant with each other. Table 1 summarizes most of the "independent" analyses of such data, listing the authors (column 1), the emission lines considered (column 2), the derived time of the onset of the far side emission (column 3), the adopted/measured angular size of the ring (column 4) and, finally, the resulting distance modulus (column 5).

Table 1. Summary of SN 1987A distance determinations based on UV line light curves and HST imaging

\begin{tabular}{llccc}
\hline Authors & Emission Lines/Ions & $\begin{array}{c}t_{\max } \\
\text { days }\end{array}$ & $\begin{array}{c}\mathrm{R}^{\prime \prime} \\
\operatorname{mas}\end{array}$ & $\begin{array}{c}(\mathrm{m}-\mathrm{M}) \\
\text { SN 1987A }\end{array}$ \\
\hline \multicolumn{2}{c}{ Prelim. IUE reductions } & & & \\
Panagia et al. 1991 [8] & $\mathrm{NIII}]$, NIV], NV, CIII] & $413 \pm 24$ & $825 \pm 17$ & $18.55 \pm 0.13$ \\
Gould 1995 [2] & $\mathrm{NIII}]$, NIV] & $390 \pm 2$ & $858 \pm 11$ & $18.35 \pm 0.04$ \\
$\quad$ Final IUE reductions & & & & \\
Sonneborn et al. 1997 [14 & $\mathrm{NIII}]$ & $399 \pm 15$ & $858 \pm 11$ & $18.43 \pm 0.10$ \\
Gould \& Uza 1998 [3] & $\mathrm{NIII}]$, NIV] & $378 \pm 5$ & $858 \pm 11$ & $18.37 \pm 0.04$ \\
Panagia et al. 2003 [9] & $\mathrm{NIII}]$, CIII], OIII] & $395 \pm 5$ & $808 \pm 17$ & $18.55 \pm 0.05$ \\
\hline
\end{tabular}


One sees immediately that most of the discrepancy can be attributed to the different angular size adopted and/or to the selection of UV emission lines that were employed to estimate the absolute size.

In particular, the "high" value of the angular size, 858 mas, is the average of the sizes measured by [10] on FOC images taken mostly with the [OIII] filter between August 1990 and October 1993. Since the apparent size of the ring increases with time, such an average represent a gross overestimate (about $6 \%$ ) of the ring size at the time of the UV maximum which leads to an underestimate of the distance modulus of 0.13 magnitudes: this effect alone accounts for most of the discrepancies.

The second point to consider is the time of the far side emission onset, $t_{\max }$. As mentioned in Sec. 22 light curves of different ions give different values of $t_{\text {max }}$. This is due to both measurement uncertainties and physical effects, such as:

- different ions recombine at different rates;

- different lines react faster or slower to a general temperature decline, i.e. cooling, depending on their excitation potential;

- the ring is made of a multitude of condensations with a wide range of densities and temperatures, with the effect that intrinsic, and possibly large fluctuations add on top of the measurement errors to distort the average behaviour of light curves.

To minimize these effect one has to combine the results of as many light curves as possible but selecting only of ions with similar characteristics, which is is what we have done in our study.

The conclusion is that all apparent discrepancies can be explained in terms of less-than-perfect selections of the data to compare with each other.

Our geometric determination of the LMC distance modulus is in excellent agreement with the recent determinations by Romaniello et al. [12] that are based on a study of both Red Clump stars and TRGB stars measured in multiband HST images of SN 1987A field. In particular, they obtained $(m-M)_{R C}=$ $18.59 \pm 0.04 \pm 0.08$ and $(m-M)_{T R G B}=18.69 \pm 0.25 \pm 0.06$ (the quoted errors are the statistical and systematic ones, respectively), whose weighted average is $<(m-M)>_{\text {LMC field }}=18.60 \pm 0.04 \pm 0.08$.

It is apparent that the true LMC distance modulus must be around 18.60 and that values lower than 18.48 and than higher 18.72 are to be excluded with high confidence.

The main consequence of our distance determination is that all Cepheid distances based on the canonical value of 18.50 for the $\operatorname{LMC}(e . g$. . 7]) should be increased by about $3 \%$. And, of course, all values of $H_{0}$ based directly or indirectly on Cepheid distances should be reduced by the same amount.

In this light, I like to assess the consequences for the determination of $H_{0}$ based on a Cepheid calibration of the peak brightness of type Ia supernovae (SNIa) relatively nearby (up tp $\sim 25 \mathrm{Mpc}$ ) and comparison of Hubble diagrams of more distant SNIa. In a long term HST project led by Sandage, Saha 
and Tammann, 9 SNIa in spiral galaxies have been calibrated with Cepheid variables, resulting in average absolute magnitudes for type Ia supernovae $M_{\mathrm{B}}=-19.47 \pm 0.07$ and $M_{\mathrm{V}}=-19.46 \pm 0.06$ with the assumption of a LMC distance modulus of 18.50 [13]. Entering these values into the Hubble diagram of more distant SNIa leads to values of the Hubble constant around $H_{0}=61 \pm 6 \mathrm{~km} \mathrm{~s}^{-1} \mathrm{Mpc}^{-1}$ for an adopted cosmological model with $\Omega_{M}=$ $0.3, \Omega_{\Lambda}=0.7$ [13].

As said above, the new LMC distance modulus would imply a reduced value of the Hubble constant, by about $-3 \%$. However, one has to take into account reddening corrections for distant supernovae in the Hubble diagram (this problem was partly bypassed in Sandage et al. analysis by considering a Hubble diagram that included only SNIa affected by little reddening) whose effect may increase the value of $H_{0}$ by as much as $+7 \%$ (see e.g. [4, 11]). Combining the two competing effects in an approximate way results in a Hubble constant of

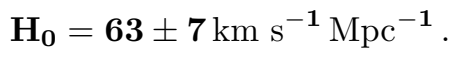

Although it is obtained with a simplified analysis which can, and will be refined, I regard this as a rather robust result that is not likely to change much in the years to come, and that offers the pleasant feature of not violating any constraint posed by old stars and the evolution of the Universe.

\section{References}

1. E. Dwek, J.E. Felten: Astrophys. J. 387, 551 (1992)

2. A. Gould: Astrophys. J. 452, 189 (1995)

3. A. Gould, O. Uza: Astrophys. J. 494, 118 (1998)

4. M. Hamuy, M.M. Phillips, N.B. Suntzeff, R.A. Schommer, J. Maza, R. Aviles: Astron. J. 112, 2391 (1996)

5. P. Jakobsen, R. Albrecht, C. Barbieri, et al.: Astrophys. J. 369, L63 (1991)

6. M. Livio, M. Donahue, N. Panagia, eds.: The Extragalactic Distance Scale, CUP (Cambridge, England) (1997)

7. B. Madore, W. Freedman: Publ. Astr. Soc. Pacific 103, 933 (1991)

8. N. Panagia, R. Gilmozzi, F. Macchetto, H.-M. Adorf, R.P. Kirshner: Astrophys. J. 380, L23 (1991); and erratum: Astrophys. J. 386, L31 (1992)

9. N. Panagia, R. Gilmozzi, R.P. Kirshner, C.S.J. Pun, G. Sonneborn: Astrophys. J., to be submitted (2003)

10. P.C. Plait, P. Lundqvist, R.A. Chevalier, R.P. Kirshner: Astrophys. J. 439, 730 (1995)

11. A.G Riess, W.H. Press, R. P. Kirshner: Astrophys. J. 473, 588 (1996)

12. M. Romaniello, M. Salaris, S. Cassisi, N. Panagia: Astrophys. J. 530, 738 (2000)

13. A. Saha, A. Sandage, G.A. Tammann, A.E. Dolphin, J. Christensen, N. Panagia, F.D. Macchetto: Astrophys. J. 562, 314 (2001)

14. G. Sonneborn, C. Fransson, P. Lundqvist, et al.: Astrophys. J. 477, 848 (1997)

15. G. Sonneborn, C.S.J. Pun, R.A. Kimble, et al.: Astrophys. J. 492, L139 (1998)

16. R. van der Marel, M.-R.L Cioni: : Astron. J. 122, 1807 (2001) 